\title{
Properties of a Generalized Divergence Related to Tsallis Relative Entropy
}

\author{
Rui F. Vigelis, Luiza H.F. de Andrade, Charles C. Cavalcante, Senior Member, IEEE,
}

\begin{abstract}
In this paper, we investigate the partition inequality, joint convexity, and Pinsker's inequality, for a divergence that generalizes the Tsallis Relative Entropy and Kullback-Leibler divergence. The generalized divergence is defined in terms of a deformed exponential function, which replaces the Tsallis $q$-exponential. We also constructed a family of probability distributions related to the generalized divergence. We found necessary and sufficient conditions for the partition inequality to be satisfied. A sufficient condition for the joint convexity was established. We proved that the generalized divergence satisfies the partition inequality, and is jointly convex, if, and only if, it coincides with the Tsallis relative entropy. As an application of partition inequality, a criterion for the Pinsker's inequality was found.
\end{abstract}

\section{Index Terms}

Kullback-Leibler divergence, Tsallis relative entropy, generalized divergence, family of probability distributions, partition inequality, joint convexity, Pinsker's inequality.

\section{INTRODUCTION}

Statistical divergences play an essential role in Information Theory [1]. Divergence can be interpreted as a measure of dissimilarity between two probability distributions. Applications that use it span from areas such as communications to econometric and other physical systems [1]. Entropy can be derived from the notion of divergence. Numerous definitions of divergence can be found in the literature. The interest in different statistical divergences is motivated by applications related to optimization and statistical learning, since more flexible functions and expressions may be suitable for larger classes of data and signals, leading to more efficient information recovery methods [2], [3], [4]. The divergence usefulness depends on its properties, such as non negativity, monotonicity and joint convexity, among others.

The counterpart of Shannon entropy is the well-known Kullback-Leibler $(\mathrm{KL})$ divergence [5], denoted by $D_{\mathrm{KL}}(\cdot \| \cdot)$, which is extensively used in Information Theory. Tsallis relative entropy $D_{q}(\cdot \| \cdot)$, which generalizes KL divergence, is defined in terms of the $q$-logarithm [6], [7]. Both KL divergence and Tsallis relative entropy satisfy some important properties, such as non negativity, joint convexity, and Pinsker's inequality [8], [9], [10]. A generalized divergence $D_{\varphi}(\cdot \| \cdot)$ can be defined in terms of a deformed exponential function $\varphi$, which plays the role of $q$-logarithm in Tsallis relative entropy. The generalized divergence appeared before in the literature, as a specific case in a broader class of divergences. Zhang in [11] introduced a divergence denoted by $D_{f, \rho}^{(\alpha)}(\cdot \| \cdot)$, where $\alpha \in[-1,1]$, and $f$ and $\rho$ are functions. The generalized divergence corresponds to Zhang's divergence with $\alpha=-1$, and $\rho=f^{-1}=\varphi^{-1}$ for a deformed exponential function $\varphi$. In [12], another class of divergences was investigated. The divergences $D_{\beta}^{c}(\cdot \| \cdot)$ in this class are given in terms of parameters $\beta=\left(\phi, M_{1}, M_{2}, M_{3}, \lambda\right)$. Expression (1) in [12], which defines $D_{\beta}^{c}(\cdot \| \cdot)$, reduces to the generalized divergence, with $\phi=-\varphi^{-1}, M_{1}=1, M_{2}=1$, $M_{3}=\left(\varphi^{-1}\right)^{\prime}(q)$, and $\lambda=\lambda_{\#}$ is the counting measure.

In [11], [12], the proposed divergences were investigated from a geometric and minimization perspectives. Some properties, which are useful in Information Theory, have not been analyzed for these divergences. In this work, we investigate the partition inequality, joint convexity, and Pinsker's inequality. We also consider the family of probability distributions associated with the generalized divergence $D_{\varphi}(\cdot \| \cdot)$. We showed necessary and sufficient conditions for the generalized divergence to satisfy the partition inequality. A sufficient condition for the joint convexity of $D_{\varphi}(\cdot \| \cdot)$ was found. We proved that $D_{\varphi}(\cdot \| \cdot)$ satisfies the partition inequality, and is jointly convex, if, and only if, it coincides with the Tsallis relative entropy $D_{q}(\cdot \| \cdot)$. Ours results for Pinsker's inequality are in accordance to previous works [13], [14].

The rest of paper is organized as follows. In Section $\amalg-A$ we provide the definition of generalized divergence. Section $\Pi-B$ is devoted to the construction of a family of probability distributions. Properties of the generalized divergence are studied in Section [III Finally, conclusions and perspectives are stated in Section IV

R. F. Vigelis is with Computer Engineering, Campus Sobral, Federal University of Ceará, 62.010-560, Sobral-CE, Brazil, e-mail:rfvigelis@ufc.br

L. H.F. Andrade is with Department of Natural Sciences, Mathematics and Statistics, Federal Rural University of the Semi-arid Region, 59.625-900, Mossoró-RN, Brazil, e-mail:luizafelix@ufersa.edu.br

C. C. Cavalcante is with Department of Teleinformatics Engineering, Federal University of Ceará, 60020-181, Fortaleza-CE, Brazil, e-mail:charles@ufc.br

Copyright (c) 2017 IEEE. Personal use of this material is permitted. However, permission to use this material for any other purposes must be obtained from the IEEE by sending a request to pubs-permissions@ieee.org. 


\section{Generalized DiverGenCE}

The generalized divergence is defined in terms of a deformed exponential function $\varphi(\cdot)$. Writing the KL divergence or Tsallis relative entropy in appropriate form, we can obtain the generalized divergence by replacing $\ln (\cdot)$ or $\ln (\cdot)$ by the inverse of a deformed exponential $\varphi^{-1}(\cdot)$. We also provide a construction of a family of probability distributions related the generalized divergence.

\section{A. Definitions}

For simplicity we denote the set of all probability distributions on $I_{n}=\{1, \ldots, n\}$ by

$$
\Delta_{n}=\left\{\left(p_{1}, \ldots, p_{n}\right): \sum_{i=1}^{n} p_{i}=1 \text { and } p_{i} \geq 0 \text { for all } i\right\} .
$$

The generalized divergence is defined for probability distribution in the interior of $\Delta_{n}$, which is denoted by $\Delta_{n}^{\circ}$. A probability distribution $\boldsymbol{p}=\left(p_{i}\right)$ belongs to $\Delta_{n}^{\circ}$ if and only if $p_{i}>0$ for each $i$,

A deformed exponential function is a convex function $\varphi: \mathbb{R} \rightarrow[0, \infty)$ such that $\lim _{u \rightarrow-\infty} \varphi(u)=0$ and $\lim _{u \rightarrow \infty} \varphi(u)=\infty$. It is easy to verify that the ordinary exponential and Tsallis $q$-exponential are deformed exponential functions. The Tsallis $q$-exponential $\exp _{q}: \mathbb{R} \rightarrow[0, \infty)$ is given by

$$
\exp _{q}(x)= \begin{cases}{[1+(1-q) x]_{+}^{1 /(1-q)},} & \text { if } q \in(0,1] \\ \exp (x), & \text { if } q=1,\end{cases}
$$

where $[x]_{+}=x$ for $x \geq 0$, and $=0$ otherwise. The Tsallis $q$-logarithm $\ln _{q}:(0, \infty) \rightarrow \mathbb{R}$ is defined as the inverse of exp $(\cdot)$, which is given by $\ln _{q}(x)=\frac{1}{1-q}\left(x^{1-q}-1\right)$ if $q \in(0,1]$.

Fixed a deformed exponential function $\varphi: \mathbb{R} \rightarrow[0, \infty)$, the generalized divergence (or generalized relative entropy) between two probability distributions $\boldsymbol{p}=\left(p_{i}\right)$ and $\boldsymbol{q}=\left(q_{i}\right)$ in $\Delta_{n}^{\circ}$ is defined as

$$
D_{\varphi}(\boldsymbol{p} \| \boldsymbol{q})=\sum_{i=1}^{n} \frac{\varphi^{-1}\left(p_{i}\right)-\varphi^{-1}\left(q_{i}\right)}{\left(\varphi^{-1}\right)^{\prime}\left(p_{i}\right)}
$$

Clearly, expression (1) reduces to the KL divergence $D_{\mathrm{KL}}(\boldsymbol{p} \| \boldsymbol{q})=-\sum_{i=1}^{n} p_{i} \ln \left(\frac{q_{i}}{p_{i}}\right)$ if $\varphi$ is the exponential function. Tsallis relative entropy in its standard form is given by $D_{q}(\boldsymbol{p} \| \boldsymbol{q})=-\sum_{i=1}^{n} p_{i} \ln _{q}\left(\frac{q_{i}}{p_{i}}\right)$. The equality

$$
-p_{i} \frac{\left(q_{i} / p_{i}\right)^{1-q}-1}{1-q}=\frac{1}{p_{i}^{q}}\left(\frac{p_{i}^{1-q}-1}{1-q}-\frac{q_{i}^{1-q}-1}{1-q}\right)
$$

shows that $D_{q}(\cdot \| \cdot)$ can be written as in (1) if $\varphi$ is the Tsallis $q$-exponential.

The non-negativity of $D_{\varphi}(\cdot \| \cdot)$ is a consequence of the concavity of $\varphi^{-1}(\cdot)$. Because $\varphi^{-1}(\cdot)$ is concave, it follows that

$$
(y-x)\left(\varphi^{-1}\right)^{\prime}(y) \leq \varphi^{-1}(y)-\varphi^{-1}(x), \quad \text { for all } x, y>0 .
$$

Using this inequality with $y=p_{i}$ and $x=q_{i}$, we can write

$$
D_{\varphi}(\boldsymbol{p} \| \boldsymbol{q})=\sum_{i=1}^{n} \frac{\varphi^{-1}\left(p_{i}\right)-\varphi^{-1}\left(q_{i}\right)}{\left(\varphi^{-1}\right)^{\prime}\left(p_{i}\right)} \geq \sum_{i=1}^{n}\left(p_{i}-q_{i}\right)=0 .
$$

Its is clear that $D_{\varphi}(\boldsymbol{p} \| \boldsymbol{q})=0$ if $\boldsymbol{p}=\boldsymbol{q}$. The converse depends on whether $\varphi^{-1}(x)$ is strictly concave. Indeed, if we suppose that $\varphi^{-1}(x)$ is strictly concave, then an equality in equation (2) is attained if and only if $x=y$. Therefore, when $\varphi^{-1}(x)$ is strictly concave, the equality $D_{\varphi}(\boldsymbol{p} \| \boldsymbol{q})=0$ is satisfied if and only if $\boldsymbol{p}=\boldsymbol{q}$.

In addition to similarities between the generalized divergence, KL divergence, and Tsallis relative entropy, there exists another motivation for the choice of expression given as in (1). We can associate with the generalized relative entropy $D_{\varphi}(\cdot \| \cdot)$ a $\varphi$-family of probability distributions, just as the KL divergence is related to the moment-generating function in a exponential family of probability distributions.

\section{B. Families of probability distributions}

For each probability distribution $\boldsymbol{p}=\left(p_{i}\right) \in \Delta_{n}^{\circ}$, we can define a deformed exponential family (of probability distributions) centered at $\boldsymbol{p}$. A deformed exponential family consists of a parameterization for the set $\Delta_{n}^{\circ}$. We remark that a deformed exponential family depends on the centered probability distribution $\boldsymbol{p}$. We can associate with each probability distribution $\boldsymbol{p} \in \Delta_{n}^{\circ}$ a deformed exponential family centered at $\boldsymbol{p}$. 
Assume that $\varphi: \mathbb{R} \rightarrow[0, \infty)$ is a positive, deformed exponential function with continuous derivative. Fixed $\boldsymbol{p}=\left(p_{i}\right) \in \Delta_{n}^{\circ}$, let $\boldsymbol{c}=\left(c_{i}\right)$ be a vector such that $p_{i}=\varphi\left(c_{i}\right)$ for each $i$. We also fix a vector $\boldsymbol{u}_{0}=\left(u_{0 i}\right)$ such that $u_{0 i}>0$ for each $i$, and

$$
\sum_{i=1}^{n} u_{0 i} \varphi^{\prime}\left(c_{i}\right)=1
$$

A deformed exponential family (of probability distributions) centered at $\boldsymbol{p}$ is a parameterization of $\Delta_{n}^{\circ}$, which maps each vector $\boldsymbol{u}=\left(u_{i}\right)$ in the subspace

$$
B_{c}^{\varphi}=\left\{\left(u_{1}, \ldots, u_{n}\right): \sum_{i=1}^{n} u_{i} \varphi^{\prime}\left(c_{i}\right)=0\right\}
$$

to a probability distribution $\boldsymbol{q}=\left(q_{i}\right) \in \Delta_{n}^{\circ}$ by the expression

$$
q_{i}=\varphi\left(c_{i}+u_{i}-\psi_{\boldsymbol{c}}(\boldsymbol{u}) u_{0 i}\right)
$$

where $\psi_{\boldsymbol{c}}: B_{\boldsymbol{c}}^{\varphi} \rightarrow[0, \infty)$ is the normalizing function, which is introduced so that (4) defines a probability density in $\Delta_{n}^{\circ}$.

The choice for $\boldsymbol{u} \in B_{\boldsymbol{c}}^{\varphi}$ is not arbitrary. Thanks to this choice, it is possible to find $\psi_{\boldsymbol{c}}(\boldsymbol{u}) \geq 0$ for which expression (4) is a probability density in $\Delta_{n}^{\circ}$. We will justify this claim. Because $\varphi(\cdot)$ is convex, it follows that

$$
y \varphi^{\prime}(x) \leq \varphi(x+y)-\varphi(x), \quad \text { for all } x, y \in \mathbb{R} .
$$

Using (5) with $x=c_{i}$ and $y=u_{i}$, we can write, for any $\boldsymbol{u} \in B_{c}^{\varphi}$,

$$
1=\sum_{i=1}^{n} u_{i} \varphi^{\prime}\left(c_{i}\right)+\sum_{i=1}^{n} \varphi\left(c_{i}\right) \leq \sum_{i=1}^{n} \varphi\left(c_{i}+u_{i}\right)
$$

By the definition of $\varphi(\cdot)$, the map

$$
g(\lambda)=\sum_{i=1}^{n} \varphi\left(c_{i}+u_{i}-\lambda u_{0 i}\right)
$$

is continuous, approaches 0 as $\lambda \rightarrow \infty$, and tends to $\infty$ as $\alpha \rightarrow \infty$. Since $\varphi(\cdot)$ is strictly increasing, it follows that $g(\cdot)$ is strictly decreasing. Then we can conclude that there exists a unique $\lambda_{0}=\psi_{\boldsymbol{c}}(\boldsymbol{u}) \geq 0$ for which $q_{i}=\varphi\left(c_{i}+u_{i}-\lambda_{0} u_{0 i}\right)$ is a probability distribution in $\Delta_{n}^{\circ}$.

The generalized divergence $D_{\varphi}(\cdot \| \cdot)$ is associated with the deformed exponential family (4) by the equality

$$
\psi_{\boldsymbol{c}}(\boldsymbol{u})=D_{\varphi}(\boldsymbol{p} \| \boldsymbol{q})=\sum_{i=1}^{n} \frac{\varphi^{-1}\left(p_{i}\right)-\varphi^{-1}\left(q_{i}\right)}{\left(\varphi^{-1}\right)^{\prime}\left(p_{i}\right)} .
$$

Using $\sum_{i=1}^{n} u_{i} \varphi^{\prime}\left(c_{i}\right)=0$, together with the constraint (3), we can write

$$
\psi_{\boldsymbol{c}}(\boldsymbol{u})=\sum_{i=1}^{n}\left(-u_{i}+\psi_{\boldsymbol{c}}(\boldsymbol{u}) u_{0 i}\right) \varphi^{\prime}\left(c_{i}\right) .
$$

It is clear that

$$
-u_{i}+\psi_{\boldsymbol{c}}(\boldsymbol{u}) u_{0 i}=\varphi^{-1}\left(p_{i}\right)-\varphi^{-1}\left(q_{i}\right),
$$

and

$$
\varphi^{\prime}\left(c_{i}\right)=\frac{1}{\left(\varphi^{-1}\right)^{\prime}\left(p_{i}\right)} .
$$

Inserting (8) and (9) into (7), we obtain (6).

If $\varphi$ is the exponential function, and $u_{0 i}=1$, the deformed exponential family reduces to the well known exponential family:

$$
q_{i}=\exp \left(u_{i}-K_{\boldsymbol{p}}(\boldsymbol{u})\right) \cdot p_{i},
$$

where $K_{\boldsymbol{p}}(\boldsymbol{u})$ is the cumulant-generating function, which equals the normalizing function $\psi_{\boldsymbol{c}}(\boldsymbol{u})$.

\section{Properties of THE Generalized DiVERGENCE}

The KL divergence and Tsallis relative entropy satisfy the partition inequality, and are jointly convex. They also satisfy Pinsker's inequality. We will investigate under what conditions these properties hold for the generalized divergence. Throughout this section we assume that $\left(\varphi^{-1}\right)^{\prime \prime}(x)$ is continuous and $>0$. 


\section{A. Partition inequality}

Partition inequality, which is a case of the data processing inequality, will be used in the proof of Pinsker's inequality. Let $\mathcal{A}=\left\{A_{1}, \ldots, A_{k}\right\}$ be a partition of $I_{n}=\{1, \ldots, n\}$, i.e., $\mathcal{A}$ is a collection of subsets $A_{j} \subseteq I_{n}$ such that $A_{i} \cap A_{j}=\emptyset$, for $i \neq j$, and $\bigcup_{j=1}^{k} A_{j}=I_{n}$. For any probability distribution $\boldsymbol{p}=\left(p_{i}\right)$, we define the probability distribution $\boldsymbol{p}^{\mathcal{A}}=\left(p_{j}^{\mathcal{A}}\right)$ as

$$
p_{j}^{\mathcal{A}}=\sum_{i \in A_{j}} p_{i}, \quad \text { for each } j=1, \ldots, k .
$$

The next result gives a necessary and sufficient condition for the partition inequality to be satisfied.

Proposition 1. For the divergence $D_{\varphi}(\cdot \| \cdot)$ to satisfy the partition inequality

$$
D_{\varphi}(\boldsymbol{p} \| \boldsymbol{q}) \geq D_{\varphi}\left(\boldsymbol{p}^{\mathcal{A}} \| \boldsymbol{q}^{\mathcal{A}}\right)
$$

for all probability distributions $\boldsymbol{p}=\left(p_{i}\right)$ and $\boldsymbol{q}=\left(q_{i}\right)$, and any partition $\mathcal{A}$ of $I_{n}$, it is necessary and sufficient that the function $g=-\frac{\left(\varphi^{-1}\right)^{\prime}}{\left(\varphi^{-1}\right)^{\prime \prime}}$ be superadditive, i.e., the inequality

$$
g(x+y) \geq g(x)+g(y),
$$

be satisfied for all $x, y \in(0,1)$ such that $x+y \in(0,1)$.

The proof of Proposition 1 requires some preliminary results which are presented in the sequel.

Lemma 2. Fix any $\alpha \in(0,1)$. The mapping

$$
F_{\alpha}(x, y)=\varphi\left((1-\alpha) \varphi^{-1}(x)+\alpha \varphi^{-1}(y)\right),
$$

is superadditive in $(0,1) \times(0,1)$ if, and only if,

$$
G(x, y)=\varphi^{-1}(\varphi(x)+\varphi(y))
$$

is convex in $\left\{(x, y) \in \mathbb{R}^{2}: \varphi(x)+\varphi(y) \in(0,1)\right\}$.

Proof: Let $x_{i}, y_{i} \in(0,1)$ be such that $x_{1}+x_{2} \in(0,1)$ and $y_{1}+y_{2} \in(0,1)$. The superadditivity of $F_{\alpha}$ implies that

$$
\begin{aligned}
\varphi\left((1-\alpha) \varphi^{-1}\left(x_{1}+x_{2}\right)+\alpha \varphi^{-1}\left(y_{1}+y_{2}\right)\right) & \\
& \geq \varphi\left((1-\alpha) \varphi^{-1}\left(x_{1}\right)+\alpha \varphi^{-1}\left(y_{1}\right)\right)
\end{aligned}
$$$$
+\varphi\left((1-\alpha) \varphi^{-1}\left(x_{2}\right)+\alpha \varphi^{-1}\left(y_{2}\right)\right) .
$$

Denote $s_{i}=\varphi^{-1}\left(x_{i}\right)$ and $t_{i}=\varphi^{-1}\left(y_{i}\right)$ for $i=1,2$. Thus inequality equation (13) is equivalent to

$$
(1-\alpha) \varphi^{-1}\left(\varphi\left(s_{1}\right)+\varphi\left(s_{2}\right)\right)+\alpha \varphi^{-1}\left(\varphi\left(t_{1}\right)+\varphi\left(t_{2}\right)\right)
$$

$$
\geq \varphi^{-1}\left[\varphi\left((1-\alpha) s_{1}+\alpha t_{1}\right)+\varphi\left((1-\alpha) s_{2}+\alpha t_{2}\right)\right]
$$

which shows the desired result.

Lemma 3. The function $G$, as defined in Lemma 2 is convex if and only if $g=-\frac{\left(\varphi^{-1}\right)^{\prime}}{\left(\varphi^{-1}\right)^{\prime \prime}}$ is superadditive in $(0,1)$.

Proof: For the function $G$ to be convex, it is necessary and sufficient that its Hessian $H_{G}$ be positive semi-definitive, which is equivalent to $\operatorname{tr}\left(H_{G}\right) \geq 0$ and $J_{G}=\operatorname{det}\left(H_{G}\right) \geq 0$, where $\operatorname{tr}(\cdot)$ denotes the trace of a matrix and $\operatorname{det}(\cdot)$ is the determinant of a matrix (see [15]). Letting $z=\varphi(x)+\varphi(y)$, we can express

$$
\begin{aligned}
& \frac{\partial^{2} G}{\partial x^{2}}(x, y)=\varphi^{\prime \prime}(x)\left(\varphi^{-1}\right)^{\prime}(z)+\left[\varphi^{\prime}(x)\right]^{2}\left(\varphi^{-1}\right)^{\prime \prime}(z), \\
& \frac{\partial^{2} G}{\partial y^{2}}(x, y)=\varphi^{\prime \prime}(y)\left(\varphi^{-1}\right)^{\prime}(z)+\left[\varphi^{\prime}(y)\right]^{2}\left(\varphi^{-1}\right)^{\prime \prime}(z),
\end{aligned}
$$

and

$$
\frac{\partial^{2} G}{\partial x \partial y}(x, y)=\varphi^{\prime}(x) \varphi^{\prime}(y)\left(\varphi^{-1}\right)^{\prime \prime}(z) .
$$

If we divide the right-hand side of (14) by $-\varphi^{\prime \prime}(x)\left(\varphi^{-1}\right)^{\prime \prime}(z) \geq 0$, and we use

$$
\frac{\left[\varphi(x)^{\prime}\right]^{2}}{\varphi(x)^{\prime \prime}}=-\frac{\left(\varphi^{-1}\right)^{\prime}(\varphi(x))}{\left(\varphi^{-1}\right)^{\prime \prime}(\varphi(x))}
$$


into the resulting expression, we obtain

$$
-\frac{\left(\varphi^{-1}\right)^{\prime}(z)}{\left(\varphi^{-1}\right)^{\prime \prime}(z)}+\frac{\left(\varphi^{-1}\right)^{\prime}(\varphi(x))}{\left(\varphi^{-1}\right)^{\prime \prime}(\varphi(x))}=g(z)-g(\varphi(x)) .
$$

As a result, we conclude that $\partial^{2} G / \partial x^{2} \geq 0$ (and similarly $\partial^{2} G / \partial y^{2} \geq 0$ ) if $g$ is superadditive. Using expressions (14)-16) for the partial derivatives of $G$, we find

$$
J_{G}(x, y)=\left(\varphi^{-1}\right)^{\prime}(z)\left(\varphi^{-1}\right)^{\prime \prime}(z) \varphi^{\prime \prime}(x) \varphi^{\prime \prime}(y)
$$

$$
\left\{\frac{\left(\varphi^{-1}\right)^{\prime}(z)}{\left(\varphi^{-1}\right)^{\prime \prime}(z)}+\frac{\left[\varphi^{\prime}(y)\right]^{2}}{\varphi^{\prime \prime}(y)}+\frac{\left[\varphi^{\prime}(x)\right]^{2}}{\varphi^{\prime \prime}(x)}\right\} .
$$

In view of (17), it follows that $J_{G}(x, y) \geq 0$ is equivalent to $g(z) \geq g(\varphi(x))+g(\varphi(y))$. Thus $G$ is convex if and only if $g$ is superadditive in $(0,1)$.

Remark 4. Similar versions of these lemmas appeared previously in the literature (see [16, sec. 3.16] and [17]). The hypothesis in these versions was weaker, or just one direction was proved.

Now, we may proceed to the proof of the main result in this section.

Proof of Proposition 17. Sufficiency. Lemmas 2 and 3 imply that

$$
F_{\alpha}(x, y)=\varphi\left((1-\alpha) \varphi^{-1}(x)+\alpha \varphi^{-1}(y)\right),
$$

is superadditive in $(0,1) \times(0,1)$, for each $\alpha \in(0,1)$. Considering $\mathcal{A}=\left\{A_{1}, \ldots, A_{k}\right\}$, we denote $p_{j}^{\mathcal{A}}=\sum_{i \in A_{j}} p_{i}$ and $q_{j}^{\mathcal{A}}=\sum_{i \in A_{j}} q_{i}$. By the superadditivity of $F_{\alpha}(x, y)$, we can write

$$
\frac{1}{1-\alpha} \sum_{i=1}^{n}\left[p_{i}-F_{\alpha}\left(q_{i}, p_{i}\right)\right]
$$

$$
\geq \frac{1}{1-\alpha} \sum_{j=1}^{k}\left[p_{j}^{\mathcal{A}}-F_{\alpha}\left(q_{j}^{\mathcal{A}}, p_{j}^{\mathcal{A}}\right)\right]
$$

An application of L'Hôpital's rule on the limit below provides

$$
\begin{aligned}
\lim _{\alpha \uparrow 1} \frac{y-F_{\alpha}(x, y)}{1-\alpha} & =\lim _{\alpha \uparrow 1} \frac{y-\varphi\left((1-\alpha) \varphi^{-1}(x)+\alpha \varphi^{-1}(y)\right)}{1-\alpha} \\
& =\varphi^{\prime}\left(\varphi^{-1}(y)\right)\left[-\varphi^{-1}(x)+\varphi^{-1}(y)\right] \\
& =\frac{\varphi^{-1}(y)-\varphi^{-1}(x)}{\left(\varphi^{-1}\right)^{\prime}(y)} .
\end{aligned}
$$

Thus, in the limit $\alpha \uparrow 1$, expression (18) becomes

$$
D_{\varphi}(\boldsymbol{p} \| \boldsymbol{q}) \geq D_{\varphi}\left(\boldsymbol{p}^{\mathcal{A}} \| \boldsymbol{q}^{\mathcal{A}}\right)
$$

which is the asserted inequality.

Necessity. It is clear that if (11) holds for all $\boldsymbol{p}=\left(p_{i}\right), \boldsymbol{q}=\left(q_{i}\right)$, and $\mathcal{A}$, then

$$
\frac{\varphi^{-1}\left(p_{1}\right)-\varphi^{-1}\left(q_{1}\right)}{\left(\varphi^{-1}\right)^{\prime}\left(p_{1}\right)}+\frac{\varphi^{-1}\left(p_{2}\right)-\varphi^{-1}\left(q_{2}\right)}{\left(\varphi^{-1}\right)^{\prime}\left(p_{2}\right)}
$$

$$
\geq \frac{\varphi^{-1}\left(p_{1}+p_{2}\right)-\varphi^{-1}\left(q_{1}+q_{2}\right)}{\left(\varphi^{-1}\right)^{\prime}\left(p_{1}+p_{2}\right)}
$$

is satisfied for all $p_{1}, p_{2}$ and $q_{1}, q_{2}$ in $(0,1)$ such that the sums $p_{1}+p_{2}$ and $q_{1}+q_{2}$ are in $(0,1)$. Let us fix $p_{1}, p_{2} \in(0,1)$. We rewrite 19] as

$$
\frac{\varphi^{-1}\left(p_{1}\right)}{\left(\varphi^{-1}\right)^{\prime}\left(p_{1}\right)}+\frac{\varphi^{-1}\left(p_{2}\right)}{\left(\varphi^{-1}\right)^{\prime}\left(p_{2}\right)}-\frac{\varphi^{-1}\left(p_{1}+p_{2}\right)}{\left(\varphi^{-1}\right)^{\prime}\left(p_{1}+p_{2}\right)}
$$

which is satisfied if and only if the function

$$
\geq \frac{\varphi^{-1}\left(q_{1}\right)}{\left(\varphi^{-1}\right)^{\prime}\left(p_{1}\right)}+\frac{\varphi^{-1}\left(q_{2}\right)}{\left(\varphi^{-1}\right)^{\prime}\left(p_{2}\right)}-\frac{\varphi^{-1}\left(q_{1}+q_{2}\right)}{\left(\varphi^{-1}\right)^{\prime}\left(p_{1}+p_{2}\right)}
$$

$$
F\left(q_{1}, q_{2}\right)=\frac{\varphi^{-1}\left(q_{1}\right)}{\left(\varphi^{-1}\right)^{\prime}\left(p_{1}\right)}+\frac{\varphi^{-1}\left(q_{2}\right)}{\left(\varphi^{-1}\right)^{\prime}\left(p_{2}\right)}-\frac{\varphi^{-1}\left(q_{1}+q_{2}\right)}{\left(\varphi^{-1}\right)^{\prime}\left(p_{1}+p_{2}\right)}
$$


attains a global maximum at $\left(q_{1}, q_{2}\right)=\left(p_{1}, p_{2}\right)$. By a simple calculation, it can be verified that $\nabla F\left(p_{1}, p_{2}\right)=0$. Moreover, we express the determinant of the Hessian of $F$ at $\left(p_{1}, p_{2}\right)$ as

$$
\begin{aligned}
J_{F}\left(p_{1}, p_{2}\right)= & \frac{\left(\varphi^{-1}\right)^{\prime \prime}\left(p_{1}\right)}{\left(\varphi^{-1}\right)^{\prime}\left(p_{1}\right)} \frac{\left(\varphi^{-1}\right)^{\prime \prime}\left(p_{2}\right)}{\left(\varphi^{-1}\right)^{\prime}\left(p_{2}\right)} \\
& \quad-\frac{\left(\varphi^{-1}\right)^{\prime \prime}\left(p_{1}+p_{2}\right)}{\left(\varphi^{-1}\right)^{\prime}\left(p_{1}+p_{2}\right)} \frac{\left(\varphi^{-1}\right)^{\prime \prime}\left(p_{2}\right)}{\left(\varphi^{-1}\right)^{\prime}\left(p_{2}\right)} \\
& \quad-\frac{\left(\varphi^{-1}\right)^{\prime \prime}\left(p_{1}\right)}{\left(\varphi^{-1}\right)^{\prime}\left(p_{1}\right)} \frac{\left(\varphi^{-1}\right)^{\prime \prime}\left(p_{1}+p_{2}\right)}{\left(\varphi^{-1}\right)^{\prime}\left(p_{1}+p_{2}\right)} \\
= & \frac{1}{g\left(p_{1}\right)} \frac{1}{g\left(p_{2}\right)}-\frac{1}{g\left(p_{1}+p_{2}\right)}\left[\frac{1}{g\left(p_{1}\right)}+\frac{1}{g\left(p_{2}\right)}\right] .
\end{aligned}
$$

Because $J_{F}\left(p_{1}, p_{2}\right) \geq 0$, it follows that $g\left(p_{1}+p_{2}\right) \geq g\left(p_{1}\right)+g\left(p_{2}\right)$.

Remark 5. If $\varphi(x)=\exp (x)$ the function $g=-\frac{\left(\varphi^{-1}\right)^{\prime}}{\left(\varphi^{-1}\right)^{\prime \prime}}$ is the identity function which is additive, therefore superadditive.

\section{B. Joint convexity}

In this section, we find a sufficient condition for the joint convexity of $D_{\varphi}(\cdot \| \cdot)$. We also show that $D_{\varphi}(\cdot \| \cdot)$ satisfies the partition inequality, and is jointly convex, if, and only if, the deformed exponential function is a scaled and translated version of the Tsallis exponential.

The generalized divergence $D_{\varphi}(\cdot \| \cdot)$ is said to be jointly convex if the inequality

$$
D_{\varphi}\left(\lambda \boldsymbol{p}_{1}+(1-\lambda) \boldsymbol{p}_{2} \| \lambda \boldsymbol{q}_{1}+(1-\lambda) \boldsymbol{q}_{2}\right)
$$

$$
\leq \lambda D_{\varphi}\left(\boldsymbol{p}_{1} \| \boldsymbol{q}_{1}\right)+(1-\lambda) D_{\varphi}\left(\boldsymbol{p}_{2} \| \boldsymbol{q}_{2}\right)
$$

is satisfied for all probability distributions $\boldsymbol{p}_{1}, \boldsymbol{p}_{2}$ and $\boldsymbol{q}_{1}, \boldsymbol{q}_{2}$ in $\Delta_{n}^{\circ}$, and each $\lambda \in[0,1]$.

Before we find a sufficient condition for the joint convexity of $D_{\varphi}(\cdot \| \cdot)$, we show some preliminary results.

Lemma 6. The function $g=-\frac{\left(\varphi^{-1}\right)^{\prime}}{\left(\varphi^{-1}\right)^{\prime \prime}}$ is (strictly) concave if and only if $h=\frac{\varphi^{\prime}}{\varphi^{\prime \prime}}$ is (strictly) concave.

Proof: Inserting the expressions $\left(\varphi^{-1}\right)^{\prime}=1 / \varphi^{\prime}\left(\varphi^{-1}\right)$ and

$$
\left(\varphi^{-1}\right)^{\prime \prime}=-\frac{\varphi^{\prime \prime}\left(\varphi^{-1}\right) \cdot\left(\varphi^{-1}\right)^{\prime}}{\left[\varphi^{\prime}\left(\varphi^{-1}\right)\right]^{2}}
$$

into the definition of $g$, we can write

$$
g=\varphi^{\prime}\left(\varphi^{-1}\right) \frac{\varphi^{\prime}\left(\varphi^{-1}\right)}{\varphi^{\prime \prime}\left(\varphi^{-1}\right)}=\varphi^{\prime}\left(\varphi^{-1}\right) h\left(\varphi^{-1}\right)
$$

Some calculations show that

$$
g_{+}^{\prime}=1+h_{+}^{\prime}\left(\varphi^{-1}\right)
$$

where $(\cdot)_{+}^{\prime}$ denotes the right derivative. By the fact of $\varphi^{-1}$ is strictly increasing, we conclude that $g_{+}^{\prime}$ is (strictly) decreasing if and only if $h_{+}^{\prime}$ is (strictly) decreasing. As a result, for $g$ to be (strictly) concave, it is necessary and sufficient that $h$ be (strictly) concave.

Lemma 7. The function $g=-\frac{\left(\varphi^{-1}\right)^{\prime}}{\left(\varphi^{-1}\right)^{\prime \prime}}$ is concave if and only if the mapping

$$
F_{\alpha}(x, y)=\varphi\left((1-\alpha) \varphi^{-1}(x)+\alpha \varphi^{-1}(y)\right), \quad(x, y) \in \mathbb{R}^{2},
$$

is concave for each $\alpha \in(0,1)$.

Proof: Let us denote $z_{\alpha}=(1-\alpha) \varphi^{-1}(x)+\alpha \varphi^{-1}(y)$. Some calculations show that

$$
\begin{aligned}
\frac{\partial^{2} F_{\alpha}}{\partial x^{2}}(x, y)=(1-\alpha)\left(\varphi^{-1}\right)^{\prime \prime}(x) \varphi^{\prime}\left(z_{\alpha}\right) & \\
+ & {\left[(1-\alpha)\left(\varphi^{-1}\right)^{\prime}(x)\right]^{2} \varphi^{\prime \prime}\left(z_{\alpha}\right), } \\
\frac{\partial^{2} F_{\alpha}}{\partial y^{2}}(x, y)=\alpha & \left(\varphi^{-1}\right)^{\prime \prime}(y) \varphi^{\prime}\left(z_{\alpha}\right) \\
& +\left[\alpha\left(\varphi^{-1}\right)^{\prime}(y)\right]^{2} \varphi^{\prime \prime}\left(z_{\alpha}\right),
\end{aligned}
$$


and

$$
\frac{\partial^{2} F_{\alpha}}{\partial x \partial y}(x, y)=\alpha(1-\alpha)\left(\varphi^{-1}\right)^{\prime}(x)\left(\varphi^{-1}\right)^{\prime}(y) \varphi^{\prime \prime}\left(z_{\alpha}\right),
$$

which we use to find the following expression for the determinant of the Hessian of $F_{\alpha}$ at $(x, y)$ :

$$
J_{F_{\alpha}}(x, y)=\alpha(1-\alpha) \varphi^{\prime}\left(z_{\alpha}\right) \varphi^{\prime \prime}\left(z_{\alpha}\right)\left(\varphi^{-1}\right)^{\prime \prime}(x)\left(\varphi^{-1}\right)^{\prime \prime}(y)
$$

$$
\left\{\frac{\varphi^{\prime}\left(z_{\alpha}\right)}{\varphi^{\prime \prime}\left(z_{\alpha}\right)}+\alpha \frac{\left[\left(\varphi^{-1}\right)^{\prime}(y)\right]^{2}}{\left(\varphi^{-1}\right)^{\prime \prime}(y)}+(1-\alpha) \frac{\left[\left(\varphi^{-1}\right)^{\prime}(x)\right]^{2}}{\left(\varphi^{-1}\right)^{\prime \prime}(x)}\right\} \text {. }
$$

Denote $h=\varphi^{\prime} / \varphi^{\prime \prime}$. Noticing that

$$
\frac{\left[\left(\varphi^{-1}\right)^{\prime}\right]^{2}}{\left(\varphi^{-1}\right)^{\prime \prime}}=-\frac{\varphi^{\prime}\left(\varphi^{-1}\right)}{\varphi^{\prime \prime}\left(\varphi^{-1}\right)}
$$

we conclude that $J_{F_{\alpha}}(x, y) \geq 0$ is equivalent to $h\left(z_{\alpha}\right) \geq(1-\alpha) h\left(\varphi^{-1}(x)\right)+\alpha h\left(\varphi^{-1}(y)\right)$.

To show that the Hessian of $F_{\alpha}$ is negative semi-definitive, we have to verify, in addition, that its trace is non-positive. Since $h$ is concave and non-negative, we have

$$
\frac{\varphi^{\prime}\left(z_{\alpha}\right)}{\varphi^{\prime \prime}\left(z_{\alpha}\right)}-(1-\alpha) \frac{\varphi^{\prime}\left(\varphi^{-1}(x)\right)}{\varphi^{\prime \prime}\left(\varphi^{-1}(x)\right)} \geq 0 .
$$

If we insert (21) into (22), and multiply the resulting expression by $(1-\alpha) \varphi^{\prime \prime}\left(z_{\alpha}\right)\left(\varphi^{-1}\right)^{\prime \prime}(x) \leq 0$, we get

$$
\frac{\partial^{2} F_{\alpha}}{\partial x^{2}}(x, y)=(1-\alpha)\left(\varphi^{-1}\right)^{\prime \prime}(x) \varphi^{\prime}\left(z_{\alpha}\right)
$$

$$
+\left[(1-\alpha)\left(\varphi^{-1}\right)^{\prime}(x)\right]^{2} \varphi^{\prime \prime}\left(z_{\alpha}\right) \leq 0
$$

Analogously, we also have $\left(\partial^{2} F_{\alpha} / \partial x^{2}\right)(x, y) \leq 0$. Consequently, the Hessian of $F_{\alpha}$ has a negative trace.

From Lemma 6, it follows that $g$ is concave if and only if $F_{\alpha}$ is concave for each $\alpha \in(0,1)$.

Proposition 8. If the function $g=-\frac{\left(\varphi^{-1}\right)^{\prime}}{\left(\varphi^{-1}\right)^{\prime \prime}}$ is concave, then the divergence $D_{\varphi}(\cdot \| \cdot)$ is jointly convex.

Proof: According to Lemma 7 the mapping

$$
F_{\alpha}(x, y)=\varphi\left((1-\alpha) \varphi^{-1}(x)+\alpha \varphi^{-1}(y)\right), \quad(x, y) \in \mathbb{R}^{2},
$$

is concave for each $\alpha \in(0,1)$. Fixed an arbitrary $\boldsymbol{p}_{j}=\left(p_{j i}\right)$ and $\boldsymbol{q}_{j}=\left(q_{j i}\right)$ in $\Delta_{n}^{\circ}$ for $j=0,1$, define

$$
\begin{aligned}
& \boldsymbol{p}_{\lambda}=(1-\lambda) \boldsymbol{p}_{0}+\lambda \boldsymbol{p}_{1}, \\
& \boldsymbol{q}_{\lambda}=(1-\lambda) \boldsymbol{q}_{0}+\lambda \boldsymbol{q}_{1},
\end{aligned}
$$

for each $\lambda \in(0,1)$. Hence we can write

$$
\begin{aligned}
& \frac{1}{1-\alpha} \sum_{i=1}^{n}\left[p_{\lambda i}-F_{\alpha}\left(q_{\lambda i}, p_{\lambda i}\right)\right] \\
& \leq(1-\lambda) \frac{1}{1-\alpha} \sum_{i=1}^{n}\left[p_{0 i}-F_{\alpha}\left(q_{0 i}, p_{0 i}\right)\right] \\
& +\lambda \frac{1}{1-\alpha} \sum_{i=1}^{n}\left[p_{1 i}-F_{\alpha}\left(q_{1 i}, p_{1 i}\right)\right] .
\end{aligned}
$$

Using L'Hôpital's rule in the limit below, we obtain

$$
\begin{aligned}
\lim _{\alpha \uparrow 1} \frac{y-F_{\alpha}(x, y)}{1-\alpha} & =\lim _{\alpha \uparrow 1} \frac{y-\varphi\left((1-\alpha) \varphi^{-1}(x)+\alpha \varphi^{-1}(y)\right)}{1-\alpha} \\
& =\varphi^{\prime}\left(\varphi^{-1}(y)\right)\left[-\varphi^{-1}(x)+\varphi^{-1}(y)\right] \\
& =\frac{\varphi^{-1}(y)-\varphi^{-1}(x)}{\left(\varphi^{-1}\right)^{\prime}(y)} .
\end{aligned}
$$

Thus, in the limit $\alpha \uparrow 1$, expression 23 becomes

$$
D_{\varphi}\left(\boldsymbol{p}_{\lambda} \| \boldsymbol{q}_{\lambda}\right) \leq(1-\lambda) D_{\varphi}\left(\boldsymbol{p}_{0} \| \boldsymbol{q}_{0}\right)+\lambda D_{\varphi}\left(\boldsymbol{p}_{1} \| \boldsymbol{q}_{1}\right),
$$

which is the desired result. 
The next result is a partial converse of Proposition 8

Lemma 9. If the divergence $D_{\varphi}(\cdot \| \cdot)$ is jointly convex for some $n \geq 3$, then the function $g=-\frac{\left(\varphi^{-1}\right)^{\prime}}{\left(\varphi^{-1}\right)^{\prime \prime}}$ satisfies the inequality

$$
g\left(\frac{x+y}{2}\right) \geq \frac{g(x)+g(y)}{2}
$$

for all $x, y \in(0,1)$ such that $x+y \in(0,1)$.

Proof: If $\boldsymbol{p}_{1}=\left(p_{1 i}\right), \boldsymbol{p}_{2}=\left(p_{2 i}\right)$ and $\boldsymbol{q}_{1}=\left(q_{1 i}\right), \boldsymbol{q}_{2}=\left(q_{2 i}\right)$ then inequality (20) is equivalent to

$$
\begin{aligned}
& \sum_{i=1}^{n}\left[\lambda \frac{\varphi^{-1}\left(p_{1 i}\right)}{\left(\varphi^{-1}\right)^{\prime}\left(p_{1 i}\right)}+(1-\lambda) \frac{\varphi^{-1}\left(p_{2 i}\right)}{\left(\varphi^{-1}\right)^{\prime}\left(p_{2 i}\right)}\right. \\
&\left.-\frac{\varphi^{-1}\left(\lambda p_{1 i}+(1-\lambda) p_{2 i}\right)}{\left(\varphi^{-1}\right)^{\prime}\left(\lambda p_{1 i}+(1-\lambda) p_{2 i}\right)}\right] \\
& \geq \sum_{i=1}^{n}\left[\lambda \frac{\varphi^{-1}\left(q_{1 i}\right)}{\left(\varphi^{-1}\right)^{\prime}\left(p_{1 i}\right)}+(1-\lambda) \frac{\varphi^{-1}\left(q_{2 i}\right)}{\left(\varphi^{-1}\right)^{\prime}\left(p_{2 i}\right)}\right.
\end{aligned}
$$$$
\left.-\frac{\varphi^{-1}\left(\lambda q_{1 i}+(1-\lambda) q_{2 i}\right)}{\left(\varphi^{-1}\right)^{\prime}\left(\lambda p_{1 i}+(1-\lambda) p_{2 i}\right)}\right]
$$

For the fixed probability distributions

$$
\begin{aligned}
& \boldsymbol{p}_{1}=\left(p_{1}, p_{2}, p, p_{13}, \ldots, p_{1 n}\right), \\
& \boldsymbol{p}_{2}=\left(p_{2}, p_{1}, p, p_{23}, \ldots, p_{2 n}\right),
\end{aligned}
$$

in $\Delta_{n}^{\circ}$, we consider

$$
\begin{aligned}
& \boldsymbol{q}_{1}=\left(p_{1}+x, p_{2}+y, p-x-y, p_{13}, \ldots, p_{1 n}\right), \\
& \boldsymbol{q}_{2}=\left(p_{2}+y, p_{1}+x, p-x-y, p_{23}, \ldots, p_{2 n}\right),
\end{aligned}
$$

where $x$ and $y$ are taken so that $\boldsymbol{q}_{1}$ and $\boldsymbol{q}_{2}$ are in $\Delta_{n}^{\circ}$. Inserting these probability distributions into $(25)$ with $\lambda=1 / 2$, we can infer that the function

$$
\begin{aligned}
F(x, y)=\frac{1}{2} \frac{\varphi^{-1}\left(p_{1}+x\right)}{\left(\varphi^{-1}\right)^{\prime}\left(p_{1}\right)}+\frac{1}{2} \frac{\varphi^{-1}\left(p_{2}+y\right)}{\left(\varphi^{-1}\right)^{\prime}\left(p_{2}\right)} & \begin{aligned}
-\frac{\varphi^{-1}\left(\frac{1}{2}\left(p_{1}+x\right)+\frac{1}{2}\left(p_{2}+y\right)\right)}{\left(\varphi^{-1}\right)^{\prime}\left(\frac{1}{2} p_{1}+\frac{1}{2} p_{2}\right)}+ & \frac{1}{2} \frac{\varphi^{-1}\left(p_{2}+y\right)}{\left(\varphi^{-1}\right)^{\prime}\left(p_{2}\right)} \\
& +\frac{1}{2} \frac{\varphi^{-1}\left(p_{1}+x\right)}{\left(\varphi^{-1}\right)^{\prime}\left(p_{1}\right)}-\frac{\varphi^{-1}\left(\frac{1}{2}\left(p_{2}+y\right)+\frac{1}{2}\left(p_{1}+x\right)\right)}{\left(\varphi^{-1}\right)^{\prime}\left(\frac{1}{2} p_{2}+\frac{1}{2} p_{1}\right)}
\end{aligned}
\end{aligned}
$$

attains a global maximum at $(x, y)=(0,0)$. Further, we can also write

$$
\begin{aligned}
J_{F}(0,0)=\left[\frac{1}{g\left(p_{1}\right)}-\frac{1}{2} \frac{1}{g\left(\frac{1}{2} p_{1}+\frac{1}{2} p_{2}\right)}\right] & \cdot\left[\frac{1}{g\left(p_{2}\right)}-\frac{1}{2} \frac{1}{g\left(\frac{1}{2} p_{1}+\frac{1}{2} p_{2}\right)}\right]-\left[\frac{1}{2} \frac{1}{g\left(\frac{1}{2} p_{1}+\frac{1}{2} p_{2}\right)}\right]^{2} \\
& =\frac{1}{g\left(p_{1}\right)} \frac{1}{g\left(p_{2}\right)}-\frac{1}{g\left(\frac{1}{2} p_{1}+\frac{1}{2} p_{2}\right)}\left[\frac{1}{2} \frac{1}{g\left(p_{2}\right)}+\frac{1}{2} \frac{1}{g\left(p_{1}\right)}\right],
\end{aligned}
$$

where $J_{F}(0,0)$ is the determinant of the Hessian of $F$ at $(0,0)$. Since $F(x, y)$ attains a maximum at $(0,0)$, inequality $J_{F}(0,0) \geq 0$ implies $g\left(\frac{1}{2} p_{1}+\frac{1}{2} p_{2}\right) \geq \frac{1}{2} g\left(p_{1}\right)+\frac{1}{2} g\left(p_{2}\right)$.

Proposition 10. Assume that $n \geq 3$. Then the generalized divergence $D_{\varphi}(\cdot \| \cdot)$ satisfies the partition inequality, and is jointly convex, if, and only if,

$$
\varphi^{-1}(x)=b \ln _{q}(x)-a, \quad \text { for } x \in(0,1),
$$

for some $q>0$ and $b>0, a \in \mathbb{R}$.

Proof: Clearly, inequalities (12) and (24) are satisfied for all $x, y \in(0,1]$. Therefore, the function $g(x)$ is superadditive and concave for $x \in(0,1 / 2)$. It is easy to verify that $g(0+)=0$. To see this, we apply the limit $x \downarrow 0$ in $0 \leq g(x) \leq g(x+y)-g(y)$, and use the continuity of $g$ at $y$. In addition, because $g(x)$ is concave with $g(0+)=0$, the function $g(x)$ is also subadditive 
for $x \in(0,1 / 2)$. Making $y \downarrow 0$ in $g(\lambda x+(1-\lambda) y) \geq \lambda g(x)+(1-\lambda) g(y)$, we obtain that $g(\lambda x) \geq \lambda g(x)$ for $\lambda \in[0,1]$. From the inequalities $g(x) \geq \frac{x}{x+y} g(x+y)$ and $g(y) \geq \frac{y}{x+y} g(x+y)$, it follows that $g(x)+g(y) \geq g(x+y)$. Hence we conclude that $g(x)$ is additive for $x \in(0,1 / 2)$.

By [18, Theorem 13.5.2], there exists $q>0$ such that $g(x)=x / q$ for $x \in(0,1 / 2)$. Using (12), and letting $y \downarrow 0$ in (24), we get

$$
g(x) \geq g\left(\frac{x}{2}\right)+g\left(\frac{x}{2}\right), \quad \text { and } \quad g\left(\frac{x}{2}\right) \geq \frac{g(x)}{2},
$$

which imply $g(x)=2 g(x / 2)$ for all $x \in(0,1)$. Hence, expression $g(x)=x / q$ is also verified for $x \in(0,1)$. Solving

$$
g(x)=-\frac{\left(\varphi^{-1}\right)^{\prime}(x)}{\left(\varphi^{-1}\right)^{\prime \prime}(x)}=\frac{x}{q}
$$

with respect to $\varphi^{-1}(x)$, we find $b>0$ and $a \in \mathbb{R}$ such that

$$
\begin{aligned}
\varphi^{-1}(x) & =b \frac{x^{1-q}}{1-q}-a \\
& =b \ln _{q}(x)-a, \quad \text { for } q \neq 1,
\end{aligned}
$$

and

$$
\varphi^{-1}(x)=b \ln (x)-a, \quad \text { for } q=1,
$$

for every $x \in(0,1)$.

The converse direction follows from Propositions 1 and 8 .

\section{Pinsker's inequality}

Pinsker's inequality relates the divergence with the $\ell_{1}$-distance. This inequality implies that convergence in divergence is stronger than convergence in the $\ell_{1}$-distance For the KL divergence, Pinsker's inequality is given by

$$
D_{\mathrm{KL}}(\boldsymbol{p} \| \boldsymbol{q}) \geq \frac{1}{2}\|\boldsymbol{p}-\boldsymbol{q}\|_{1}^{2},
$$

where $\|\boldsymbol{p}-\boldsymbol{q}\|_{1}=\sum_{i=1}^{n}\left|p_{i}-q_{i}\right|$ is the $\ell_{1}$-distance between probability distributions $\boldsymbol{p}=\left(p_{i}\right)$ and $\boldsymbol{q}=\left(q_{i}\right)$ in $\Delta_{n}^{\circ}$.

The next result shows Pinsker's inequality for the generalized divergence.

Theorem 11 (Pinsker's Inequality). Suppose that the partition inequality (11) holds. In addition, assume that

$$
c=\inf _{0<p<q<1} \frac{1}{8} \frac{1}{q-p}\left[-\frac{\left(\varphi^{-1}\right)^{\prime}(q)}{\left(\varphi^{-1}\right)^{\prime}(p)}+\frac{\left(\varphi^{-1}\right)^{\prime}(1-q)}{\left(\varphi^{-1}\right)^{\prime}(1-p)}\right]>0 .
$$

Then, for any probability distributions $\boldsymbol{p}=\left(p_{i}\right)$ and $\boldsymbol{q}=\left(q_{i}\right)$ in $\Delta_{n}^{\circ}$, the generalized divergence satisfies the inequality

$$
D_{\varphi}(\boldsymbol{p} \| \boldsymbol{q}) \geq c\|\boldsymbol{p}-\boldsymbol{q}\|_{1}^{2} .
$$

Proof: Let $\mathcal{A}=\left\{A_{1}, A_{2}\right\}$ be a partition of $I_{n}$, where $A_{1}=\left\{i: p_{i} \geq q_{i}\right\}$ and $A_{2}=\left\{i: p_{i}<q_{i}\right\}$. Hence we can write

$$
\begin{aligned}
\|\boldsymbol{p}-\boldsymbol{q}\|_{1} & =\sum_{i=1}^{n}\left|p_{i}-q_{i}\right| \\
& =\sum_{i \in A_{1}}\left(p_{i}-q_{i}\right)+\sum_{i \in A_{2}}\left(q_{i}-p_{i}\right) \\
& =\left(p_{1}^{\mathcal{A}}-q_{1}^{\mathcal{A}}\right)+\left(q_{2}^{\mathcal{A}}-p_{2}^{\mathcal{A}}\right) \\
& =\left\|\boldsymbol{p}^{\mathcal{A}}-\boldsymbol{q}^{\mathcal{A}}\right\|_{1} .
\end{aligned}
$$

By the partition inequality

$$
D_{\varphi}(\boldsymbol{p} \| \boldsymbol{q}) \geq D_{\varphi}\left(\boldsymbol{p}^{\mathcal{A}} \| \boldsymbol{q}^{\mathcal{A}}\right)
$$

we see that it suffices to show

$$
D_{\varphi}\left(\boldsymbol{p}^{\mathcal{A}} \| \boldsymbol{q}^{\mathcal{A}}\right) \geq c\left\|\boldsymbol{p}^{\mathcal{A}}-\boldsymbol{q}^{\mathcal{A}}\right\|_{1}^{2} .
$$

Let us denote $p_{1}^{\mathcal{A}}=p$ and $q_{1}^{\mathcal{A}}=q$. Then inequality (33) can be rewritten as

$$
\frac{\varphi^{-1}(p)-\varphi^{-1}(q)}{\left(\varphi^{-1}\right)^{\prime}(p)}+\frac{\varphi^{-1}(1-p)-\varphi^{-1}(1-q)}{\left(\varphi^{-1}\right)^{\prime}(1-p)} \geq 4 c(p-q)^{2},
$$


since $\left\|\boldsymbol{p}^{\mathcal{A}}-\boldsymbol{q}^{\mathcal{A}}\right\|_{1}=2(p-q)$. For a fixed $p \in(0,1)$, we define the function

$$
F(q)=\frac{\varphi^{-1}(p)-\varphi^{-1}(q)}{\left(\varphi^{-1}\right)^{\prime}(p)}
$$

$$
+\frac{\varphi^{-1}(1-p)-\varphi^{-1}(1-q)}{\left(\varphi^{-1}\right)^{\prime}(1-p)}-4 c(p-q)^{2}
$$

for $q \in(0,1)$. By the symmetry of the terms $p$ and $q$ in $[31$, it is clear that

$$
c=\inf _{0<q<p<1} \frac{1}{8} \frac{1}{q-p}\left[-\frac{\left(\varphi^{-1}\right)^{\prime}(q)}{\left(\varphi^{-1}\right)^{\prime}(p)}+\frac{\left(\varphi^{-1}\right)^{\prime}(1-q)}{\left(\varphi^{-1}\right)^{\prime}(1-p)}\right]>0 .
$$

As a result, the derivative

$$
F^{\prime}(q)=(q-p)\left\{\frac{1}{q-p}\left[-\frac{\left(\varphi^{-1}\right)^{\prime}(q)}{\left(\varphi^{-1}\right)^{\prime}(p)}+\frac{\left(\varphi^{-1}\right)^{\prime}(1-q)}{\left(\varphi^{-1}\right)^{\prime}(1-p)}\right]-8 c\right\}
$$

is $\geq 0$ for $q>p$, and $\leq 0$ for $q<p$. We conclude that $F(q)$ attains a minimum at $q=p$. Therefore,

$$
D_{\varphi}\left(\boldsymbol{p}^{\mathcal{A}} \| \boldsymbol{q}^{\mathcal{A}}\right)-c\left\|\boldsymbol{p}^{\mathcal{A}}-\boldsymbol{q}^{\mathcal{A}}\right\|_{1}^{2}=F(q) \geq F(p)=0,
$$

and inequality $(32)$ follows.

If we assume $\varphi^{-1}(x)=\log (x)$, then expression (31) results in $c=1 / 2$, which is the constant in Pinsker's inequality for the KL divergence. For the Tsallis exponential, an easy computation shows that $c=q / 2$ in equation (31) with $\varphi^{-1}(x)=\ln _{q}(x)$. This result is in accordance to the work of Gilardoni [13], which investigated the Pinsker's inequality for $f$-divergences. Gilardoni showed that the $f$-divergence $D_{f}(\boldsymbol{p} \| \boldsymbol{q})=\sum_{i=1}^{n} p_{i} f\left(\frac{q_{i}}{p_{i}}\right)$ satisfies the inequality $D_{f}(\boldsymbol{p} \| \boldsymbol{q}) \geq \frac{f^{\prime \prime}(1)}{2}\|\boldsymbol{p}-\boldsymbol{q}\|_{1}^{2}$, supposing that $f$ is convex and three times differentiable at $x=1$ with $f^{\prime \prime}(1)>0$. Tsallis relative entropy is an $f$-divergence with $f(x)=-\ln _{q}(x)$. In this case, we have $f^{\prime \prime}(1)=q$.

\section{CONCLusions}

In this work, we found necessary and sufficient conditions for the generalized divergence $D_{\varphi}(\cdot \| \cdot)$ to satisfy the partition inequality. We also showed a condition that implies the joint convexity of $D_{\varphi}(\cdot \| \cdot)$. It was proved that, for the generalized divergence $D_{\varphi}(\cdot \| \cdot)$ to coincide with the Tsallis relative entropy $D_{q}(\cdot \| \cdot)$, it is necessary and sufficient that $D_{\varphi}(\cdot \| \cdot)$ satisfy the partition inequality, and be jointly convex. As an application of partition inequality, a criterion for the Pinsker's inequality was found. We also constructed a family of probability distributions associated with the generalized divergence.

This work can be extended in many aspects. The data processing inequality was not proved. Comparisons between generalized divergences, as investigated in [19] for $f$-divergences, have the potential of being a prosperous topic of research. In [20], a generalization of Rényi divergence was defined in terms of a deformed exponential. As future work, we aim to investigate the properties of this generalized Rényi divergence.

\section{ACKNOWLEDGMENT}

The authors would like to thank CNPq (Procs. 408609/2016-8 and 309472/2017-2) and Coordenação de Aperfeiçoamento de Pessoal de Nível Superior - Brazil (CAPES) - Finance Code 001 for partial funding of this research. We would also like to thank Sueli I.R. Costa for the valuable contributions to this work.

\section{REFERENCES}

[1] T. M. Cover and J. A. Thomas, Elements of information theory, 2nd ed. Wiley-Interscience [John Wiley \& Sons], Hoboken, NJ, 2006.

[2] T. Hastie, R. Tibshirani, and J. Friedman, The elements of statistical learning, 2nd ed., ser. Springer Series in Statistics. Springer, New York, 2009, data mining, inference, and prediction.

[3] J. C. Principe, Information theoretic learning, ser. Information Science and Statistics. Springer, New York, 2010, Rényi’s entropy and kernel perspectives.

[4] S. Konishi and G. Kitagawa, Information criteria and statistical modeling, ser. Springer Series in Statistics. Springer, New York, 2008.

[5] S. Kullback and R. A. Leibler, "On information and sufficiency," Ann. Math. Statistics, vol. 22, pp. 79-86, 1951. [Online]. Available: https://doi.org/10.1214/aoms/1177729694

[6] L. Borland, A. R. Plastino, and C. Tsallis, "Information gain within nonextensive thermostatistics," J. Math. Phys., vol. 39, no. 12, pp. 6490-6501, 1998. [Online]. Available: https://doi.org/10.1063/1.532660

[7] _ , "Erratum: "Information gain within generalized [non-extensive] thermostatistics"," J. Math. Phys., vol. 40, no. 4, p. 2196, 1999. [Online]. Available: https://doi.org/10.1063/1.533119

[8] T. van Erven and P. Harremoës, "Rényi divergence and Kullback-Leibler divergence," IEEE Trans. Inform. Theory, vol. 60, no. 7, pp. 3797-3820, 2014. [Online]. Available: https://doi.org/10.1109/TIT.2014.2320500

[9] S. Furuichi, K. Yanagi, and K. Kuriyama, "Fundamental properties of Tsallis relative entropy," J. Math. Phys., vol. 45, no. 12, pp. 4868-4877, 2004.

[10] S. Furuichi, "On uniqueness theorems for Tsallis entropy and Tsallis relative entropy," IEEE Trans. Inform. Theory, vol. 51, no. 10, pp. 3638-3645, 2005. [Online]. Available: https://doi.org/10.1109/TIT.2005.855606

[11] J. Zhang, "Divergence function, duality, and convex analysis," Neural Comput., vol. 16, no. 1, pp. 159-195, Jan. 2004

[12] M. Broniatowski and W. Stummer, "Some universal insights on divergences for statistics, machine learning and artificial intelligence," in Geometric structures of information, ser. Signals Commun. Technol. Springer, Cham, 2019, pp. 149-211. 
[13] G. L. Gilardoni, “On Pinsker's and Vajda's type inequalities for Csiszár's f-divergences,” IEEE Trans. Inform. Theory, vol. 56, no. 11, pp. 5377-5386, 2010.

[14] I. Sason and S. Verdú, " $f$-divergence inequalities," IEEE Trans. Inform. Theory, vol. 62, no. 11, pp. 5973-6006, 2016. [Online]. Available: https://doi.org/10.1109/TIT.2016.2603151

[15] R. Bhatia, Positive definite matrices, ser. Princeton Series in Applied Mathematics. Princeton University Press, Princeton, NJ, 2007.

[16] G. H. Hardy, J. E. Littlewood, and G. Pólya, Inequalities, ser. Cambridge Mathematical Library. Cambridge University Press, Cambridge, 1988, reprint of the 1952 edition.

[17] J. Matkowski, "The converse of the Minkowski's inequality theorem and its generalization," Proc. Amer. Math. Soc., vol. 109, no. 3, pp. 663-675, 1990. [Online]. Available: https://doi.org/10.2307/2048205

[18] M. Kuczma, An introduction to the theory of functional equations and inequalities, 2nd ed. Birkhäuser Verlag, Basel, 2009.

[19] P. Harremoës and I. Vajda, "On pairs of $f$-divergences and their joint range," IEEE Trans. Inform. Theory, vol. 57, no. 6, pp. 3230-3235, 2011. [Online]. Available: https://doi.org/10.1109/TIT.2011.2137353

[20] D. C. de Souza, R. F. Vigelis, and C. C. Cavalcante, "Geometry induced by a generalization of Rényi divergence," Entropy, vol. 18, no. 11, pp. Paper No. 407, 16, 2016. [Online]. Available: https://doi.org/10.3390/e18110407

Rui F. Vigelis received the B.Sc degree in Electrical Engineering from the Federal University of Ceará, Brazil, in 2005, and the M.Sc and Ph.D. degrees in Teleinformatics Engineering from the Federal University of Ceará, Brazil, in 2006 and 2011, respectively. Since 2012, he is an Assistant Professor at the Federal University of Ceará, campus Sobral. His primary research interests are in the analysis of non-standard function spaces (e.g., Musielak-Orlicz spaces), non-parametric information geometry, and measures of information.

Luiza H.F. Andrade received the B.Sc degree in Mathematics from the Ceará State University, in 2002, the M.Sc degree in Mathematics and the Ph.D. degree in Teleinformatics Engineering, both from the Federal University of Ceará, in 2007 and 2018, respectively. She has been developing research in information geometry and information theory.

Charles C. Cavalcante (S'98 - M'04 - SM'11) received the B.Sc and M.Sc in Electrical Engineering from the Federal University of Ceará (UFC), Brazil, in 1999 and 2001, respectively, and the Ph.D. degree from the University of Campinas (UNICAMP), Brazil, in 2004. He has held a grant for Scientific and Technological Development from 2004 to 2007 and since March 2009 he has a grant of Scientific Research Productivity both from the Brazilian Research Council (CNPq). He is now an Associate Professor at Teleinformatics Engineering Department of UFC holding the Statistical Signal Processing chair. From August 2014 to July 2015 he was a Visiting Assistant Professor at the Department of Computer Science and Electrical Engineering (CSEE) from University of Maryland, Baltimore County (UMBC) in the United States. He has been working on signal processing strategies for communications where he has several papers published in journal and conferences, has authored three international patents and he has worked on several funded research projects on the signal processing and wireless communications areas. He is also a co-author of the book Unsupervised Signal Processing: Channel Equalization and Source Separation and co-editor of the book Signals and Images: Advances and Results in Speech, Estimation, Compression, Recognition, Filtering, and Processing, both published by CRC Press. He is a researcher of the Wireless Telecommunications Research Group (GTEL) where he leads research on signal processing and wireless communications. Dr. Cavalcante is a Senior Member of the IEEE and Senior Member of the Brazilian Telecommunications Society (SBrT) for the term 2018-2020. Since March 2018 he is the President of the Brazilian Telecommunication Society (SBrT) and has just been elected to the IEEE Signal Processing Society Board of Governors in the capacity of Regional Director-at-Large for Regions 7 \& 9 for the term 2020-2021. His main research interests are in signal processing for communications, statistical signal processing and information geometry. 